\title{
Patient reported outcome measures in practice
}

Scores of tools to measure outcomes that matter to patients have been developed over the past 30 years but few are used routinely at the point of care. Nelson and colleagues describe examples where they are used in primary and secondary care and argue for their wider uptake to improve quality of care

\section{Eugene C Nelson professor ${ }^{12}$, Elena Eftimovska researcher ${ }^{3}$, Cristin Lind patient advocate ${ }^{4}$, Andreas Hager patient advocate ${ }^{56}$, John $\mathrm{H}$ Wasson professor $^{1}$, Staffan Lindblad professor ${ }^{37}$}

\begin{abstract}
${ }^{1}$ Dartmouth Institute for Health Policy and Clinical Practice, Geisel School of Medicine, Dartmouth College, 35 Centerra Parkway, Suite 300, Lebanon, NH 03766, USA; ${ }^{2}$ Dartmouth-Hitchcock Health, Lebanon, NH, USA ; ${ }^{3}$ Department of Learning, Informatics, Management and Ethics, Karolinska Institutet, Sweden ; ${ }^{4}$ Rare Diseases Sweden, Stockholm, Sweden ; ${ }^{5}$ Genia, Stockholm, Sweden ; ${ }^{6}$ Hansen Law, Stockholm, Sweden ; ${ }^{7}$ Quality Register Centre, Stockholm, Sweden
\end{abstract}

Clinicians' understanding of the effect of disease and treatment on patients' daily lives is poor. ${ }^{1}$ In response to this problem, over the past three decades, hundreds of standardised measures have been developed to capture patient reported outcomes, including symptom status, physical function, mental health, social function, and wellbeing. However, the patient reported outcome measures (PROMs) movement has largely been driven by the agenda of researchers or service payers and has failed to focus effectively on improving the quality of care from the patient's perspective (box 1). We use two examples to show how the use of PROMs in everyday practice has the potential to narrow the gap between the clinician's and patient's view of clinical reality and help tailor treatment plans to meet the patient's preferences and needs. ${ }^{2}$

\section{Barriers to routine use}

Evidence shows that the systematic use of information from PROMs leads to better communication and decision making between doctors and patients and improves patient satisfaction with care..$^{3-7}$ There is also evidence that patients report better outcomes - for example, improvement in depression. ${ }^{8}$ However, research on attempts to embed measurement of patient reported outcomes into routine practice has revealed many technical, social, cultural, legal, and logistical barriers to successful adoption. $^{9-13}$

Clinicians are often reluctant to use PROMs routinely because they fear it will add to their workload rather than make them more efficient and effective. Furthermore, many clinicians who do spend time talking to patients contend that they already understand their patients' problems and do not need additional information from them.
Patients generally welcome systems that routinely use PROMs. However, they say that patient reporting systems must be used well and not misdirect the focus of the clinical encounter, burden patients, or focus only on factors that have value to clinicians (box 2).

Any system designed to assess and respond to patient reported outcomes must include relevant and validated measures. These must be analysed and reported appropriately and the response to them made explicit in the notes. Identifying the best way to incorporate PROMs in pre-existing medical record systems while safeguarding privacy is challenging. The best design will vary for different patient populations, practice types, and clinical settings.

Below we present two cases from Sweden and the United States that show routine use of PROMs in primary and secondary care. Both systems were developed by experienced, practising physicians and share similar objectives but the design differs.

\section{Swedish rheumatology quality registry}

The Swedish rheumatology quality registry was established in 1995 and contains data on over 66000 patients, about $85 \%$ of people in Sweden with rheumatoid arthritis. ${ }^{14}$ Data generated by patients is fed into a user friendly "dashboard" and used to support care. Patient reported outcome measures are tracked over time in relation to ongoing treatment. Patients input information on both disease specific and general measures of function and health, helping clinicians to provide more appropriate and patient centred care. The dashboard displays trends and is used to guide treatment, shared decision making, and self management. Experience suggests that the registry 


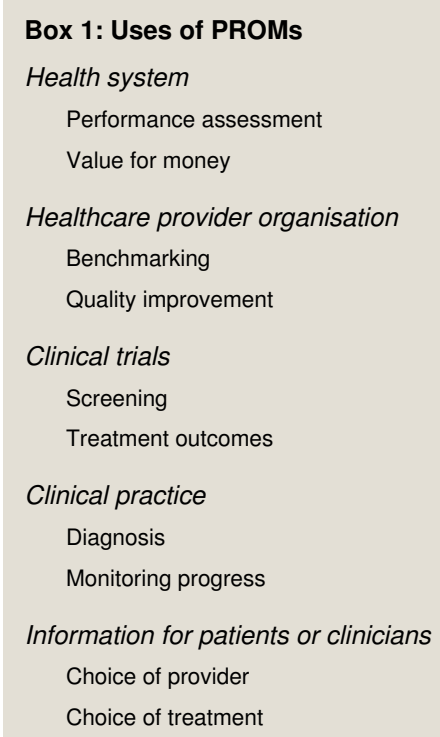

\section{Box 2: Views of patient advocates}

For most patients there is no systematic or effective method for communicating what happens outside the clinical encounter, such as perceived needs, symptoms, response to treatment, undesirable side effects, effect on function, and what matters to patients and their families. Like clinicians, patients want better outcomes for individuals and communities, and better professional development and system performance, although we might not use those same words to describe them.

PROM systems have the potential to enable improvement by providing information that can bridge the gap between the clinical reality and the patient world, triggering learning as well as the right next action.

PROM systems must be codeveloped by patients, the public, and professionals to obtain maximum value. They should be integrated with the rest of the patients' healthcare information and patients should be able to use the information when and where they choose, including for research to benefit others with their condition.

dashboard helps engage and empower patients and increases their confidence that they can manage and control their condition. $^{14}$

Karin A, who has rheumatoid arthritis, says: "Nowadays I work together with my doctor and the computer for every change in my treatment. I can check my disease at home between visits. Before I go to see my doctor I report on and measure my own health. It's easy; I just click on the screen and enter how I feel now."

Clinical outcome measures seem to improve after patients start measuring, reporting, and sharing responsibility for the management of their condition with their physician. Patients in one region (Gävle County) that implemented routine use of PROMs in clinic visits had less disease activity, as measured by $\mathrm{C}$ reactive protein, than patients in regions where structured communication with doctors was not necessarily part of routine care. $^{15}$

Patients have a personal identifier to access the registry, and the system is easy for patients to use and input information. The system also contains all the usual data used by medical staff to track outcomes (blood test results, inflamed joint counts, etc) so staff do not need to spend extra time inputting data or to move from one screen to another. Because patients track their outcomes between office visits and can communicate electronically when there is a change, the timing of appointments (urgent care for flare-up or routine follow-up) and their duration and content can be preplanned by the office staff and guided by patients' needs and priorities. Registry data can also be aggregated to examine population health, improve quality of care and provide transparent public reports on patient outcomes at the county level.

\section{US primary care model: HowsYourHealth.org}

Developed in 1994 and disseminated on the internet since 1999 without charge to primary care practices, the HowsYourHealth system provides an immediate and standard source of information about patients' function, diagnosis, symptoms, health habits, preventive needs, capacity to self manage chronic conditions, and their experiences of care. ${ }^{16}$ Patients enter all the data themselves, mainly using tick boxes in response to questions. For practice improvement the system also provides a summary of all patient data (with national benchmarks) and a secure registry to target interventions at groups of patients with similar needs (such as those with diabetes, emotional problems, or low confidence to self manage health problems). The system is used by hundreds of practices in the United States and Canada, and initiatives to increase uptake are under way in Iowa, Massachusetts, and British Columbia. The system is endorsed by several medical specialty organisations.

Patients usually access HowsYourHealth from home or within the doctor's office. They have the opportunity to share their "check-up" data with the office staff, receive information tailored to their needs, and create a personal health plan for tracking and sharing their health status and behaviours. They can also access tools to help build confidence and skill in self management and better understand the risks and benefits of treatment. The system collects generic patient reported outcomes, including COOP/WONCA measures on pain, mobility, mental health, age and sex specific preventive health needs, and self management capacity for common risks and conditions. Patients control their Hows YourHealth information and its security, and it doesn't require a personal identifier or password. 
A controlled trial involving 45 primary care physicians and 1651 patients aged $\geq 70$ years found that the HowsYourHealth system significantly improved patient ratings of overall care quality and their understanding of important risks (such as falls and advance planning of care). Patients also reported that it helped with daily activities, emotional issues, and social support. ${ }^{7}$ Another controlled trial involving 47 primary care physicians and 644 adults with pain and emotional problems showed sustained improvement when Hows YourHealth was combined with a problem solving intervention supported by a nurse educator. ${ }^{8}$

Practices typically build Hows YourHealth into the routine "annual check-up," and patients are usually happy to use it once they understand how it helps their care and improve the practice. James Bloomer, a family practitioner, describes his practice's approach:

"Our practice routinely asks all patients to complete Hows YourHealth as part of their check-up. The tool often brings to light important concerns that we are unaware of, such as domestic abuse, and we spend no time actively collecting these data."

Empirical observations from practices are used to expand knowledge about implementation and customisation. For example, experience has shown that using patient volunteers rather than practice staff to implement HowsYourHealth is often more successful.

\section{Other examples of PROMs in practice}

In England standard patient reported outcome measures are being used to track the outcomes of patients with four common surgical conditions. ${ }^{17}{ }^{18}$ In the Netherlands, care teams are using web based applications and mobile health to monitor the quality of life of children with cancer. ${ }^{19}$ In the United States, over 60 practices in the ImproveCareNow network are using patient reported outcomes and clinical data to improve results for children with inflammatory bowel disease. Since establishing the network in 2007, remission rates have improved from 55\% to $77 \%$ for 17000 patients, in 30 states. $^{20}$

\section{Looking to the future}

Emerging information and communication technologies are spurring the development of new systems designed to capture and use the patient voice. However, the extent to which these systems can improve care will depend on how effectively clinicians respond to the patient voice. ${ }^{78}$ Future systems for incorporating the patient voice and patient reported outcomes into office practice need to be based on fundamental principles of patient centred care such as the timely explanation of information based on patient need and delivery of the information in a way that can help patients take effective actions to protect their health and wellbeing.
We are grateful for the contributions of James Bloomer, Angela Coulter, Dave deBronkart, Andy Gage, Sara Riggare, Rosemund Snow, Sven Tegmark, James Weinstein, Rupert Whitaker, and Paul Wicks.

Competing interests: We have read and understood BMJ policy on declaration of interests and declare ECN owns stock in Quality Data Management, JHW under licence with the Trustees of Dartmouth College develops HowsYourHealth.org and related websites and distributes them without compensation to primary care practices, and $\mathrm{AH}$ is a patient support system entrepreneur, patient advocate, and caregiver to a child with cystic fibrosis.

Provenance and peer review: Commissioned; externally peer reviewed.

1 Nelson EC, Conger B, Douglass R, Gephart D, Kirk J, Page R, et al. Functional health status levels of primary care patients. JAMA 1983:249:3331-8.

2 Barry M, Edgman-Levitan S. Shared decision making-the pinnacle of patient-centered care. N Engl J Med 2012;366:780-1.

3 Chen J, Ou L, Hollis SJ. A systematic review of the impact of routine collection of patient reported outcome measures on patients, providers and health organisations in an oncologic setting. BMC Health Serv Res 2013;13:211.

4 Marshall S, Haywood K, Fitzpatrick R. Impact of patient-reported outcome measures on routine practice: a structured review. J Eval Clin Pract 2006;12:559-68.

5 Santana M-J, Feeny D. Framework to assess the effects of using patient-reported outcome measures in chronic care management. Qual Life Res 2014;23:1505-13.

6 Valderas JM, Kotzeva A, Espallargues M, Guyatt G, Ferrans CE, Halyard MD, et al. The impact of measuring patient-reported outcomes in clinical practice: a systematic review of the literature. Qual Life Res 2008;17:179-93.

7 Wasson JH, Stukel TA, Weiss JE, Hays RD, Jette AM, Nelson EC. A randomized trial of using patient self-assessment data to improve community practices. Effect Clin Pract 1999;2:1-10.

8 Ahles TA, Wasson JW, Seville JL, Johnson DJ, Cole BF, Hanscom B, et al. A controlled trial of methods for managing pain in primary care patients with or without co-occurring psychosocial problems. Ann Fam Med 2006;4:341-50

9 Dawson J, Doll H, Fitzpatrick R, Jenkins C, Carr AJ. The routine use of patient reported outcome measures in healthcare settings. BMJ 2010;340:c186.

10 Nelson EC, Fisher ES, Weinstein JN. A perspective on patient-centric, feed-forward "collaboratories." In: Institute of Medicine. Engineering a learning healthcare system: a look at the future: workshop summary. National Academies Press, 2011:149-70.

11 Nelson EC, Hvitfeldt H, Reed R, Grossman D, Lindblad S, Mastanduno M, et al. Using patient-reported information to improve health outcomes and health care value: case studies from Dartmouth, Karolinska and Group Health. 2012. http://tdi.dartmouth.edu/ images/uploads/tdi_tr_pri_ia_sm.pdf.

12 Pincus T, Summey JA, Soraci SA, Jr, Walston K, Hummon N. Assessment of patient satisfaction in activities of daily living using a modified Stanford Health Assessment Questionnaire. Arthritis Rheum 1983;26:1346-53.

13 Taylor W, Wang MC, Lozano P, Bradford SC. Patient reported data to engage patients and inform clinical and population care: using patient reported outcome data to provide patient-centered care and improve clinical care delivery. National Quality Conference, Sacramento, CA, 4 June 2013.

14 Hvitfeldt H, Carli C, Nelson EC, Mortenson DM, Ruppert BA, Lindblad S. Feed forward systems for patient participation and provider support: adoption results from the original US context to Sweden and beyond. Qual Manag Health Care 2009:18:247-56.

15 Svensk Reumatologis Kvalitetsregister. Årsrapport 2012 [Swedish Rheumatology Quality Registry's Annual report 2012 ]. 2013. http://srq.nu/srqny/wp-content/uploads/2014/04/\% C3\%85rsrapport-2012.pdf.

16 Wasson JH, Benjamin R, Johnson D, Moore LG, Mackenzie T. Patients use the internet to enter the medical home. J Amb Care Mgmt 2011;34:38-46.

17 Devlin NJ, Appleby J. Getting the most out of PROMs: putting health outcomes at the heart of NHS decision-making [Internet]. Health Economics. 2010. Available from: http:// www.kingsfund.org.uk/publications/proms.html.

18 Devlin NJ, Parkin D, Browne J. Patient-reported outcome measures in the NHS: new methods for analysing and reporting EQ-5D data. Health Econ 2010;19:886-905.

19 Haverman L, Engelen V, van Rossum MA, Heymans HS, Grootenhuis MA. Monitoring health-related quality of life in paediatric practice: development of an innovative web-based application. BMC Pediatr 2011:11:3.

20 Crandall W, Kappelman CW, Colletti RB, Leibowitz I, Grunow JE, Ali S, et al. ImproveCareNow: the development of a pediatric inflammatory bowel disease improvement network. Inflamm Bowel Dis 2011;17:450-7.

Cite this as: BMJ 2015;350:g7818

(c) BMJ Publishing Group Ltd 2015 